\title{
POTENCIALES EVOCADOS VISUALES EN CUIDADOS INTENSIVOS
}

Jesús H. Rodríguez, MD* Ángela Gómez, MD** Raúl Corredor, MD *** Clara López de Meza.****

\section{Resumen}

El objeto de este trabajo es comprobar si los potenciales evocados visuales en los pacientes hospitalizados en la unidad de cuidados intensivos (UCI) y bajo sedación varían en sus parámetros de amplitud y latencia, según se realicen con los ojos abiertos o cerrados. Se realizaron ocho potenciales evocados visuales en pacientes hospitalizados en la UCI, con alteración de su estado de conciencia por sedación farmacológica, sin compromiso neurológico; según la historia clínica y las patologías de tratamiento no eran de origen neurológico. No se encontraron diferencias en cuanto a los parámetros de amplitud y velocidad de conducción (latencia) cuando se realizaban con los ojos abiertos y cerrados. Sin embargo llama la atención la mejoría en la velocidad que oscila alrededor de $1 \%$ y que si bien clínicamente no altera el resultado final de normalidad si representa un incremento de 1,5 ms de conducción nerviosa al realizarse con los ojos cerrados. Los potenciales evocados pueden continuar realizándose sin tener en cuenta la postura de los párpados en los pacientes bajo sedación en UCI, teniendo así la certeza de que los datos obtenidos reflejan la integridad de la vía visual en sujetos que por su estado de conciencia no pueden colaborar con otros datos clínicos.

Palabras claves: potenciales evocados visuales, sedación farmacológica, vía visual.

\section{Introducción}

Basados en observaciones electrofisiológicas los potenciales evocados visuales aportan una gran información acerca de la integridad de la vía visual, en los pacientes que se encuentran con depresión de su estado de conciencia debido a que sus condiciones patológicas necesitan de soporte avanzado vital y al estar alerta interfieren con ese soporte. Los potenciales evocados visuales pueden brindar información acerca de la integridad de uno de los componentes del sistema nervioso sensible a las noxas de tipo isquémico, subsecuentes a eventos hemodinámicos o hipoxémicos, ya que se evocan áreas que con frecuencia son afectadas en situaciones de baja perfusión sanguínea cerebral. Es necesario, entonces, que se aclare si en estas situaciones influye el hecho de que la determinación de los potenciales evocados se realice con los ojos abiertos o cerrados y si

\footnotetext{
* Residente IV año, Departamento de Neurología, Fundación Universitaria de Ciencias de la Salud, Hospital de San José.

* Neurofisióloga, FSB.

*** Neurólogo, Hospital de San José.

****Asesora metodológica y estadística. Laboratorio de Neurofisiología Hospital de San José, Unidad de Cuidados Intensivos Hospital de San José., Bogotá D.C., Colombia.
}

existen variaciones en cuanto a la velocidad de neuroconducción mediante las latencias y la amplitud.

La conciencia es el estado en el cual el individuo se da cuenta de sí mismo y del ambiente, siendo el estado de coma lo opuesto y entre los dos estados existen varias etapas de la alteración del grado de conciencia. ${ }^{1}$ De acuerdo a la relación con el tiempo, se considera que las alteraciones de conciencia pueden ocurrir en forma aguda o crónica; así, los grados incluyen: ${ }^{2}$ a). Alerta y consciente, cuando el individuo se encuentra con el conocimiento de sí mismo y de su ambiente y tiene una respuesta adecuada a su ambiente o interrelación; b). Somnoliento, cuando el individuo tiende a permanecer dormido pero un estímulo débil, como el llamado, es capaz de alertarlo y entonces puede interrelacionarse con el ambiente de manera adecuada y está conciente de sí mismo y de su entorno; c). Estupor: se define como el estado de conciencia en el cual el individuo es capaz de alertarse con estímulos mayores, como el dolor o la estimulación vigorosa, sin que logre alertarse adecuadamente ni tener una interrelación adecuada con el medio ni conciencia de sí mismo; d). Coma superficial: el individuo no se alerta, pero sí realiza movimientos de 
localización a los estímulos vigorosos. e) Coma profundo: en este caso, el sujeto no se alerta ni tampoco hay respuesta motora a ningún tipo de estímulo, por más vigoroso que sea.

Por su parte, los estados crónicos de alteración de la conciencia se definen como aquellos que ocurren en individuos que, posteriores a eventos agudos, 21 días después persisten con alteraciones de la conciencia y se encuentran en las categorías de estado vegetativo crónico persistente o estado de conciencia mínimo con implicaciones legales y de pronostico según sus definiciones. $^{3}$

La vía visual en el ser humano comienza con los receptores visuales en el ojo, a saber, conos y bastones, los cuales constituyen un órgano sensorial altamente especializado que modifica los estímulos luminosos y se encuentran en mayor concentración en la fóvea. Los estímulos luminosos previamente sufren un direccionamiento desde el cristalino que enfoca los rayos luminosos hacia la retina y su intensidad es regulada por el esfínter correspondiente; luego, al llegar a la retina atraviesan sus diversas capas y alcanzan los fotorreceptores, denominados conos y bastones por la forma de sus segmentos externos.

Los bastones funcionan como parte de un sistema monocromático, mientras que los conos funcionan como receptores de color; ambos receptores son estimulados por los fotones del haz de luz en el pigmento de rodopsina, que es una combinación de la proteína opsina y el isómero cis derivados de la vitamina A. Tras la absorción de un fotón se producen cambios en la permeabilidad de membrana del fotorreceptor, que interrumpe la llamada corriente oscura y que produce la señal que es transmitida a las células intermedias horizontales y posteriormente a las células ganglionares, cuyos axones se reúnen y conforman el disco papilar y luego el nervio óptico, el cual llega hasta el quiasma. En esta estructura ocurre la decusación de las fibras provenientes de la porción temporal hacia el hemisferio cerebral contralateral, llegando en primer término al núcleo geniculado lateral dorsal del cuerpo geniculado lateral del tálamo, por medio de las cintillas ópticas. De aquí, la información es transmitida hacia la corteza visual a través de la proyección geniculoestriada o radia- ción óptica. En el hombre el área cortical visual cubre ambas paredes de la cisura calcarina posterior y porciones adyacentes del polo occipital o área 17 de Brodman. La transmisión de la información de la vía visual desde la retina es organizada topográficamente.

La estimulación de los órganos de los sentidos o de los nervios periféricos evoca una reacción de las áreas receptoras corticales apropiadas. ${ }^{5} \mathrm{Y}$ también en diversas estaciones subcorticales de relevo. Sin embargo, no se puede colocar un electrodo de registro cerca de las estaciones de relevo, ni es posible identificar potenciales minúsculos de solo unos cuantos microvoltios dentro de la actividad de fondo de tamaño mucho mayor como en el caso del electroencefalograma o en la electromiografía.

El empleo de los métodos de promediación creados por Dawson en $1954^{6}$ y el desarrollo subsiguiente de técnicas computarizadas han brindado los medios para superar estos problemas. Las ondas de latencia breve se modifican en cada estación de relevo y se registran mediante electrodos dístales " Registro de campo distante". Estas ondas se vuelven máximas en las computadoras hasta en un punto en el que se pueden medir con facilidad su latencia y su voltaje.

Una de las propiedades más notables de los potenciales evocados es su resistencia a la anestesia y los fármacos sedantes, en comparación con la actividad medida por el electroencefalograma (EEG), incluso cuando hay lesión de los hemisferios cerebrales; esto permite su empleo para vigilar la integridad de las vías cerebrales en situaciones que vuelven inútil la utilización del EEG.

Chiapa y colaboradores, además de Ho, ${ }^{7,8}$ han revisado los detalles de estas técnicas. La interpretación de los potenciales evocados aferentes tales como los visuales, los auditivos y los somatosensoriales, se basan en la latencia de la aparición de las ondas después del estímulo, las latencias entre ondas y las asimetrías en el tiempo. Se han establecido normas, pero aun es aconsejable confirmarlas en cada laboratorio. De manera característica: 2,5 a 3 desviaciones estándar por arriba de la latencia media, en cualquier medición, se considera como definición de anormalidad. 
Las amplitudes de las ondas son menos informativas. En los potenciales evocados visuales un estímulo luminoso centelleante sobre la retina inicia, a menudo, ondas discernibles sobre los lóbulos occipitales. En el EEG estas reacciones a las tasas rápidas de estimulación se conocen como reacción impulsora occipital.

En 1969, Regan y Heron ${ }^{9}$ observaron que podían producir una reacción visual evocada mediante cambios repentinos del patrón del tablero de ajedrez que se está viendo. Las reacciones producidas de esta manera, mediante la repetición rápida del patrón invertido, resultaron más fáciles de identificar y medir que las reacciones a los destellos y más sostenidas en las formas de las ondas, entre un individuo y otro. Este tipo de estímulo, aplicado primero a un ojo y a continuación al otro, podría demostrar retrasos en la conducción en las vías visuales de pacientes que habían sufrido con anterioridad una enfermedad del nervio óptico, aunque en algunos casos no hubiera signos residuales de reducción en la agudeza visual, anomalías de los campos visuales, alteraciones de la cabeza del nervio óptico o cambios en los reflejos pupilares; este procedimiento, llamado reacciones visuales evocadas de patrón cambiante (RVEPC) o potenciales visuales evocados de patrón invertido, se ha adoptado con amplitud como una de las pruebas más delicadas de las lesiones del sistema visual.

Por lo general, las anomalías de la amplitud y la duración de las RVEPC acompañan a las latencias anormalmente prolongadas, pero son difíciles de cuantificar. La latencia esperada para la polaridad positiva de las RVEPC es de casi $100 \mathrm{~m} / \mathrm{s}$. (de aquí el termino P100); la latencia de cerca de $118 \mathrm{~m} / \mathrm{s}$ o la diferencia en las latencias mayores a $9 \mathrm{~m} / \mathrm{s}$ entre los dos ojos, significa afección de un nervio óptico. La prolongación bilateral de las latencias, demostrada por la estimulación separada de cada ojo, podría deberse a lesiones en ambos nervios ópticos, en el quiasma óptico o en las vías visuales que están después del quiasma.

\section{Materiales y métodos}

El objeto de este estudio es el de comprobar si los potenciales evocados visuales en los pacientes hospitalizados en la unidad de cuidados intensivos bajo sedación, varían en sus parámetros de amplitud y latencia según se realicen con los ojos abiertos o cerrados. El tipo de estudiofue descriptivo, longitudinal y prospectivo.

Durante los meses de marzo y abril del año 2001 ingresaron a la unidad de cuidados intensivos del hospital de San José en Bogota D.C., Colombia, institución universitaria adscrita a la Fundación Universitaria de Ciencias de la Salud, ocho pacientes con alteración de su estado de conciencia por sedación con diferentes depresores del SNC, quienes fueron hospitalizados por causas no neurológicas y la razón de la necesidad de la depresión del sistema nervioso obedecía a la necesidad de soporte ventilatorio, con intubación orotraqueal y en condiciones de sedación y relajación; algunos pacientes también necesitaban soporte inótropico. A todos los pacientes se les descartó compromiso neurológico por sus antecedentes en la historia clínica y antes de entrar a la UCI los médicos de la unidad de cuidados intensivos les practicaron un examen neurológico, el cual descartó compromiso neurológico previo; una vez hospitalizados en la unidad, el neurólogo del estudio también examinó a los pacientes y además, verificó que no presentaban alteraciones visuales graves, según lo descrito en la historia clínica. Así mismo, se les practicó fundoscopia a todos los pacientes, sin que fuesen registradas alteraciones visuales graves, tales como opacidades del cristalino, lesiones focales en la retina o macula, que interfirieran el examen o que alteraran los posibles resultados.

Todos los pacientes incluidos en el estudio recibieron una evaluación electrofisiológica, realizada por una técnica experta y bajo supervisión del neurólogo, con un aparato marca Cadwell Excel plus, modelo 1997 (Estados Unidos), mediante métodos estándar y siguiendo el protocolo descrito por Ho. ${ }^{7}$ Además, se realizaron promediaciones en cada ojo y en ambos, primero con los ojos cerrados y luego abiertos (tres promediaciones de estímulos, cada una de 100 estímulos totalizando 300 estímulos). Se inició con el ojo derecho, luego con el izquierdo y por último, con ambos. Cada estímulo tenía una intensidad de 100 milisegundos.

Se realizaron todas los potenciales evocados con Goggles y luz de diodos. Se verificó en la segunda serie a cada paciente que tuviera los ojos abiertos mediante la elevación de los párpados en forma mecánica. En 
tres pacientes hubo dificultades técnicas, debido a que algunas habitaciones no presentaban aislamiento del polo a tierra y se contaminó gravemente el estudio con interferencia estática, lo cual no permitió la interpretación de los estudios.

La información se procesó en un programa estadístico-epidemiológico EPI Info. 6 CDC 1994, Excel Microsoft Windows 2000 y SPSS.

\section{Resultados}

De los cinco pacientes en los cuales se pudo llevar a cabo el estudio, la edad media fue de 49,4 años, cuatro pertenecían al sexo masculino y uno al femenino y los diagnósticos fueron diferentes (Tabla 1).

A todos los pacientes se les tuvo en soporte ventilatorio mecánico, los medicamentos depresores del estado de conciencia utilizados fueron benzodiacepinas tipo midazolam con dosis que oscilaban entre $0,03 \mathrm{mg} / \mathrm{Kg} /$ hora y $0,1 \mathrm{mg} / \mathrm{Kg} /$ hora y fentanyl $70 \mathrm{mcg} /$ h. Otros medicamentos utilizados en la UCI fueron: antibióticos, soporte inotrópico con dopamina y adrenalina, diuréticos de asa y digital.

Las mediciones entre ojos, la prueba de análisis de varianza y prueba de Duncan para la verificación de si había diferencia estadística Intergrupal e intragrupo no mostraron diferencias.

\begin{tabular}{|c|c|c|c|}
\hline \multicolumn{4}{|c|}{$\begin{array}{c}\text { Tabla I. Características } \\
\text { de los pacientes estudiados }\end{array}$} \\
\hline No & Edad (años) & Sexo & Diagnostico \\
\hline 1 & 34 & $M$ & $\begin{array}{c}\text { Herida por } \\
\text { arma de fuego } \\
\text { en cuello }\end{array}$ \\
\hline 2 & 45 & M & Sepsis abdominal \\
\hline 3 & 68 & $M$ & $\begin{array}{c}\text { Esofagogastrectomía } \\
\text { por CA }\end{array}$ \\
\hline 4 & 58 & M & $\begin{array}{c}\text { Neumonía } \\
\text { adquirida en } \\
\text { la comunidad }\end{array}$ \\
\hline 5 & 42 & $\mathrm{~F}$ & $\begin{array}{c}\text { Herida } \\
\text { cortopunzante } \\
\text { en corazón }\end{array}$ \\
\hline$M=$ & asculino & & $F=$ Femenino \\
\hline
\end{tabular}

Para los potenciales efectuados con ojos abiertos, la latencia N75 (OA N75) y la vocación en la corteza visual accesoria mínima fue de $54,100 \mathrm{~m} / \mathrm{s}$, la media fue de $86,9 \mathrm{~m} / \mathrm{s}$ y la máxima fue de $98,100 \mathrm{~m} / \mathrm{s}$. Se estableció una moda de 54,100 m/s. Dichos hallazgos se encuentran dentro de un rango de normalidad con respecto a los descritos en poblaciones sin estar bajo el efecto de sedación (Figura 1).

Con los ojos abiertos, la latencia de P100 (OA P100) y la evocación en corteza occipital primaria mínima fue de $95,900 \mathrm{~m} / \mathrm{s}$, la media fue de $109,00 \mathrm{~m} / \mathrm{s}$, la máxima fue de $116 \mathrm{~m} / \mathrm{s}$ y la moda de $112 \mathrm{~m} / \mathrm{s}$. Se continúa con la tendencia de normalidad en cuanto a los parámetros con referencia a personas despiertas y se observó cómo la evocación en corteza occipital de los potenciales evocados visuales para los casos prolongados (mayores de $115 \mathrm{~m} / \mathrm{s}$ ) se corrige disminuyendo su latencia cuando se estimula biocularmente, ${ }^{5}$ como en el caso del paciente numero 3. Ello también aplicaría para pacientes bajo sedación. En el paciente número1 también se presentó alteración en la latencia P100, con una diferencia entre ojos mayor de $9 \mathrm{~m} / \mathrm{s}$ lo cual confirma una posible alteración en el ojo derecho.

Con ojos abiertos y las amplitudes (OA Ampl.)el valor mínimo fue de $0,900 \mathrm{Uv}$., la media fue de 2,200 $U v$ y el valor máximo fue de $6,600 \mathrm{Uv}$, con una moda de 1,300 Uv. Se nota para este parámetro una tendencia a las bajas amplitudes. En siete de 15 mediciones para la amplitud, se encontró por debajo del límite de normalidad para pacientes despiertos. Solo en un caso la disminución de la amplitud fue mayor al $50 \%$, mientras

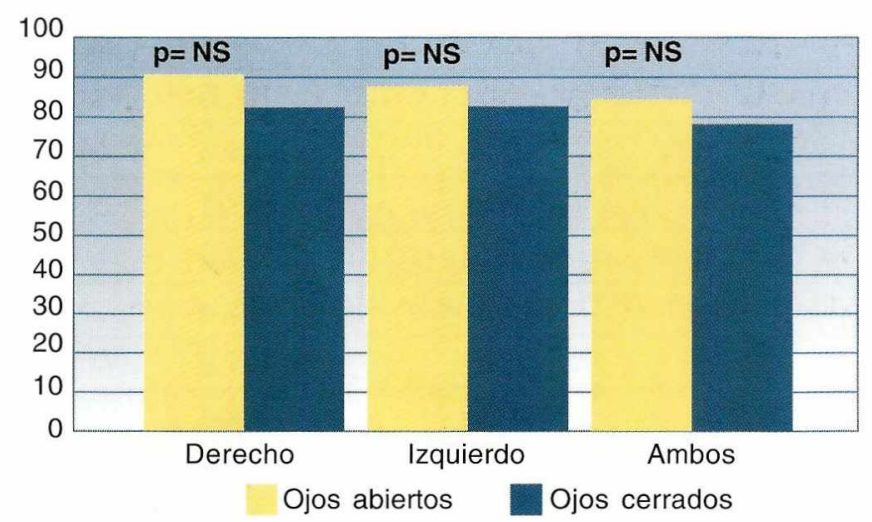

Figura I. Valores promedio de potenciales evocados visuales. Latencia N75. Corteza visual accesoria. 
que en los restantes 6 casos de disminución de la amplitud esta se encontró entre un 15\%-40\% menor.

Para los potenciales efectuados con ojos cerrados, la latencia N75 (OC N75) en la corteza visual accesoria, mínima fue de $55,000 \mathrm{~m} / \mathrm{s}$, la media fue de 85,00 $\mathrm{m} / \mathrm{s}$ y la latencia máxima fue de $97,300 \mathrm{~m} / \mathrm{s}$. Los anteriores valores se encuentran entre parámetros normales para paciente despiertos. Se anota que en el caso del paciente No 1 la diferencia entre ojos desaparece dejando de ser patológicas lo cual podría indicar una alteración local ya que se corrige al cerrar el párpado. Se estableció una moda de $80,300 \mathrm{~m} / \mathrm{s}$.

Las diferencias entre las latencias de la corteza visual accesoria con estímulo con ojos cerrados y abiertos oscilaron con una mejoría del $1,66 \%$, en cuanto a la velocidad de transmisión para el rango menor (a saber: $54,100 \mathrm{~m} / \mathrm{s}-55,000 \mathrm{~m} / \mathrm{s}$ ) y con una mejoría en la velocidad de transmisión de 1,02\% (Tabla 2).

No existieron diferencias entre la estimulación con ojos abiertos o cerrados para la latencia N75, mediante la aplicación de la prueba de anova, que no mostró diferencias significativa desde el punto de vista estadístico, aunque fisiológicamente se comprobó el aumento referido del $1 \%$ en cuanto a la velocidad, que en cifras representa $1,1 \mathrm{mts} / \mathrm{seg}$.

Con los ojos cerrados, la latencia de P100 (OC P100) y evocación en corteza occipital primaria, mínima fue de $95,400 \mathrm{~m} / \mathrm{s}$, media fue de $105,00 \mathrm{~m} / \mathrm{s}$, máxima de $114 \mathrm{~m} / \mathrm{s}$ y la moda fue de $105 \mathrm{~m} / \mathrm{s}$. Se presenta la misma tendencia a la mejoría de las velocidades de transmisión con una variación muy pequeña con respecto a las obtenidas con ojos abiertos que corresponde al $0,5 \%$ que no influye en los resultados finales de normalidad. Sin embargo aplicando la prueba de anova se documento una diferencia estadísticamente significativa para un valor de $\mathrm{P}=0,007$. Tanto para los rangos de menor y mayor tiempo de latencia. (a saber, ojos abiertos: 95,900-116 m/s a ojos cerrados: 95,400-114 m/s) (Figura 2).

La determinación de las amplitudes con los ojos cerrados mostró una mínima de $1,800 \mathrm{Uv}$, una media fue de 3,200 Uv y una máxima de 6,000 Uv, con una moda de 4,200 Uv. Con respecto a las amplitudes se presen-

Tabla 2. Hallazgos en los potenciales evocados visuales

\begin{tabular}{|c|c|c|c|c|c|c|c|}
\hline \multirow[t]{2}{*}{ Ítem No. } & \multirow[t]{2}{*}{ No. Paciente } & \multicolumn{3}{|c|}{ Ojos Abiertos } & \multicolumn{3}{|c|}{ Ojos Cerrados } \\
\hline & & $\begin{array}{l}\text { Latencia } \\
\text { N75 m/s }\end{array}$ & $\begin{array}{l}\text { Latencia } \\
\text { P } 100 \mathrm{~m} / \mathrm{s}\end{array}$ & $\begin{array}{c}\text { Amplitud } \\
U_{V}\end{array}$ & $\begin{array}{l}\text { Latencia } \\
\mathrm{N} 75 \mathrm{~m} / \mathrm{s}\end{array}$ & $\begin{array}{l}\text { Latencia } \\
\text { P } 100 \mathrm{~m} / \mathrm{s}\end{array}$ & $\begin{array}{c}\text { Amplitud } \\
U_{V}\end{array}$ \\
\hline 1 & $1 . \mathrm{OD}$ & 87,5 . & 109 & 1.30 & 55.0 & 107 & 2.80 \\
\hline 2 & OI & 55,0 & 100 & 3.80 & 57.8 & 103 & 3.70 \\
\hline 3 & Ambos & 54.1 & 99.1 & 1.70 & 58.8 & 96.3 & 3.20 \\
\hline 4 & $2 . \mathrm{OD}$ & 85.6 & 98.1 & 0.90 & 80.3 & 95.4 & 2.90 \\
\hline 5 & OI & 79.7 & 101 & 2.20 & 78.4 & 98.6 & 2.25 \\
\hline 6 & Ambos & 81.6 & 95.9 & 1.70 & 80.0 & 95.8 & 1.80 \\
\hline 7 & 3.OD & 95.3 & 116 & 1.40 & 94.6 & 110 & 2.30 \\
\hline 8 & OI & 98.1 & 112 & 1.20 & 97.3 & 109 & 2.40 \\
\hline 9 & Ambos & 90.9 & 109 & 1.30 & 89.0 & 105 & 2.00 \\
\hline 10 & 4.OD & 94.4 & 114 & 3.00 & 89.4 & 114 & 4.20 \\
\hline 11 & OI & 86.9 & 112 & 6.60 & 88.1 & 111 & 5.40 \\
\hline 12 & Ambos & 83.4 & 105 & 4.80 & 85.0 & 101 & 4.20 \\
\hline 13 & $5 . \mathrm{OD}$ & 90.4 & 115 & 5.10 & 89.6 & 110 & 6.00 \\
\hline 14 & OI & 89.6 & 112 & 4.70 & 88.0 & 109 & 5.00 \\
\hline 15 & Ambos & 82.4 & 106 & 4.90 & 80.3 & 105 & 5.10 \\
\hline
\end{tabular}


tó una mejoría de las mismas al realizarse con ojos cerrados, que con ojos abiertos y representó un crecimiento de la amplitud al $100 \%$ para los rangos mínimos y del $10 \%$ para los rangos altos.

\section{Discusión y conclusiones}

Nuestros resultados indican que no incide el hecho de realizar con ojos abiertos o cerrados los potenciales evocados visuales, en pacientes bajo sedación en las unidades de cuidados intensivos, lo cual podría aplicarse en un futuro a personas alerta y que incida en una relajación mejor lo que puede mejorar la obtención de los potenciales evocados visuales. Las diferenciäs entre un mismo individuo, para el caso del paciente No 1, fueron superiores a $9 \mathrm{~m} / \mathrm{s}$ lo cual podría demostrar una alteración ocular en dicho paciente en el ojo de mayor latencia (ojo derecho).

Existe muy poca diferencia significativa desde el punto de vista clínico con respecto a este interrogante, si bien para los demás casos se observó que la velocidad de conducción mejoró con el cierre ocular, en un porcentaje muy bajo (1\%) que no representa una diferencia clínica importante, aunque en términos fisiológicos y a velocidades de $115 \mathrm{~m} / \mathrm{s}$, equivale a $1,1 \mathrm{~m}$.

Podría concluirse que si bien los párpados interfieren en la visión, permiten el paso de la cantidad suficiente de luz para evocar una respuesta en las cortezas visuales accesorias y primarias. La amplitud disminuida en todos

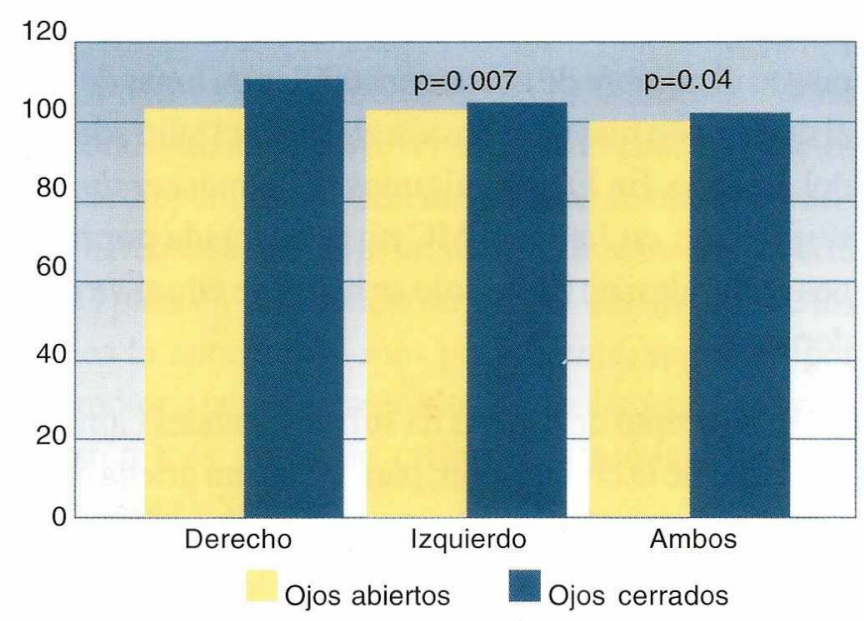

Figura 2. Valores promedio de potenciales evocados visuales. Latencia PI00. Corteza visual primaria. los casos puede corresponder a alguna alteración o interferencia, que produzcan los depresores de la conciencia en cuanto a ese parámetro de evaluación en los potenciales evocados visuales de pacientes en UCI y bajo sedación, o podría correlacionarse con una mejor transmisión de la luz, la cual evoca respuestas de más amplitud por la circunstancia de oclusión ocular; lo contrario se ha observado en los casos de pacientes con miopía.

Entonces, los potenciales evocados visuales pueden realizarse independientemente de la posición de los párpados, en los pacientes bajo sedación, hospitalizados en las unidades de cuidados intensivos, teniendo así la certeza de que los datos obtenidos reflejan la integridad de la vía visual en sujetos que, por su compromiso del estado de conciencia, no pueden colaborar con otros datos clínicos. Sin embargo, dichas conclusiones deben tomarse con reserva, debido al escaso número de pacientes evaluados, y son necesarios más estudios para corroborar o refutar tales hallazgos.

\section{Referencias}

1. Plum Fred, Estupor y Coma Segunda Edición, Manual Moderno 1982, México, DF. México. Pagina 3.

2. Diccionario de Terminología de Ciencias Medicas, $12^{a}$ Edición, Salvat Editores, 1985. Barcelona España.

3. Douglas I. Katz, M.D. MINIMALLY CONSCIOUS STATES. American Academy Of Neurology, Minneapolis, Minnesota, April 1998.

4. RHS Carpenter, Neurofisiología, Segunda Edición, Editorial Manual Moderno, México, México. 1996. Pag. 129-175.

5. Adams R. Principles of Neurology, 6ta ed Mc. Graw Hill, 1999. México, México. Trastornos de la visión, Cap. 13. Pag209225.

6. Dawson GD: A summation technique for the detection of small evoked potentials. Electroencephalogram. Clin. Neurophysiol. 6:65,1954.

7. Shin J. Ho. Clinical Electromyography nerve conductions studies. Editorial University park press. Baltimore. USA. 1999. 8. Chiappa Keith H., Evoked Potentials in Clinical Medicine, Lippincott-Raven Publishers, Philadelphia. 1997.

9. Regan D, Heron Jr: Clinical investigation of lesions of the visual pathway: A new objetive technique. J Neurol Neurosurg Psychiatry 1969. 32:479.

10. Netter F. Nervous System 1ra Ed. Masson-Salvat, 1994. Barcelona España. Tonı I/1 Pag. 171.

11. Mayo Clinic Examinations in Neurology, Seventh Edition, Editorial Mosby, Rochester, Minnesota, 1998.

12. Bruce J. Fisch. Spehlmanns EEG Primer. Tercera Edición. Editorial Elsevier, New York. NY. USA. 1999. 